\title{
Les élèves suisses à l'École des beaux-arts de Paris
} 1800-1968

\section{Marie-Laure Crosnier Leconte}

\section{(2) OpenEdition}

1 Journals

Édition électronique

URL : http://journals.openedition.org/edl/959

DOI : $10.4000 /$ edl. 959

ISSN : 2296-5084

Éditeur

Université de Lausanne

\section{Édition imprimée}

Date de publication : 15 mars 2017

Pagination : 15-30

ISBN : 978-2-940331-64-2

ISSN : 0014-2026

\section{Référence électronique}

Marie-Laure Crosnier Leconte, "Les élèves suisses à l'École des beaux-arts de Paris 1800-1968 ",

Études de lettres [En ligne], 1 | 2017, mis en ligne le 15 mars 2019, consulté le 18 décembre 2020. URL: http://journals.openedition.org/edl/959; DOI : https://doi.org/10.4000/edl.959 


\section{LES ÉLÈVES SUISSES À L'ÉCOLE DES BEAUX-ARTS DE PARIS 1800-1968}

Trois cent quarante Suisses ont été admis à l'École des beaux-arts de Paris entre 1800 et 1968. Ils ont constitué une des populations étrangères les plus significatives de l'École, avec les Américains et les Roumains, mais avec un flux de fréquentation qui se caractérise par sa régularité, avec seulement quelques pics migratoires. De quelle région venaient-ils? Quels autres établissements d'enseignement ont-ils fréquentés, tant en Suisse qu'en France? À quelle catégorie socioprofessionnelle appartenaient-ils? Quel cursus scolaire ont-ils suivi? Quels ateliers ont-ils choisi et quelle place y ont-ils occupée, ainsi qu'au sein de l'École? Quel accueil ont-ils reçu et comment eux-mêmes ont-ils accueilli les Français, notamment dans les concours publics? Une première analyse de ce corpus tente d'apporter une amorce de réponse à toutes ces questions.

Trois cent quarante élèves suisses ont été admis en architecture à l'École des beaux-arts de Paris. Ce chiffre peut paraître modeste devant la masse des élèves français qui ont constitué un contingent de plus de 18’000 élèves sur la totalité de l'histoire de l'École, entre 1800 et $1968^{1}$. Sachant que celle-ci était d'abord destinée à former les architectes français, ce

I. Voir le Dictionnaire des élèves architectes de l'École des beaux-arts (1800-1968) que nous avons constitué et mis en ligne sur le site de l'Institut national d'histoire de l'art, plateforme agorha: <http://www.purl.org/inha/agorha/001/7>. Il a été constitué par la saisie des neuf registres matricules des élèves en architecture de l'École. Enrichi du dépouillement des dossiers d'élèves, et complété des données biographiques disponibles, il a permis de dresser un corpus exhaustif de tous ceux qui ont effectué une scolarité, même embryonnaire, à l'École des beaux-arts. Il constitue une liste d'autorité à double titre: il permet de séparer ceux qui ont été scolarisés ou non à l'École des beaux-arts, mais aussi, grâce à l'extrait d'acte de naissance que les aspirants devaient fournir pour s'inscrire aux examens d'admission, d'en connaître l'identité avec précision. 
chiffre fait néanmoins des Suisses une des populations étrangères les plus représentatives de l'École, avec les Américains et les Roumains. Et, rapporté à la cohorte des quelque 700 Américains qui ont littéralement envahi l'École, essentiellement entre 1890 et 1910, il est notable que la Suisse, pays modeste par son étendue géographique et sa population, ait produit un contingent d'élèves seulement inférieur de moitié à celui des États-Unis d'Amérique.

Ce qui fait aussi la spécificité des Suisses, c'est qu'ils ont formé un flux migratoire continu.

Ils ont même eu au milieu du XVIII e siècle un pionnier en la personne du Bernois Niklaus Sprüngli (1725-1802), élève de Loriot, qui non seulement a fréquenté l'Académie royale d'architecture, mais a même été logiste au concours du Grand Prix en 1754. Seul un autre étranger, le Russe Fedor Ivanovitch Volkov, figure dans la liste des élèves de l'Académie.

Le pic de réussite à l'examen d'admission de l'École a été atteint en 1877 avec un total de 13 Suisses sur 74 élèves admis toutes nationalités confondues, dont neuf autres étrangers, ce qui constitue un succès notable.

\section{Un enseignement spécifique}

Rappelons brièvement le fonctionnement de l'enseignement à l'École des beaux-arts. Celle-ci s'est toujours posée en héritière directe des Académies royales de peinture, de sculpture et d'architecture. Délivrant un enseignement qui se voulait d'excellence à seulement quelques dizaines d'élèves destinés à se mettre au service du pouvoir, l'Académie royale d'architecture cohabitait nécessairement avec l'apprentissage en agence et les cours privés, comme l'École des Arts de Jacques François Blondel (1708/9-1774), sans oublier le système de transmission traditionnel du savoir auprès d'un maître maçon ou charpentier. La suppression des académies en 1793 n'interrompt pas l'enseignement de l'architecture, porté par une poignée d'hommes regroupés autour de Julien David Leroy (1724-1803), professeur à l'Académie d'architecture depuis 1774, qui poursuit ses cours gratuitement. Deux jeunes architectes l'accompagnent dans cette entreprise. Antoine Laurent Thomas Vaudoyer (17561846) passe pour être le premier à créer, fin 1789, un atelier dédié à la 
préparation des élèves aux concours de l'Académie royale d'architecture $^{2}$, et Charles Percier (1764-1838) fait de même sitôt revenu de son pensionnat romain fin 1791.

Le rôle de l'École des beaux-arts est d'une part de dispenser un enseignement ex cathedra, d'autre part d'organiser un ensemble de concours qui permettent d'évaluer la progression des élèves. Le nombre d'heures d'enseignement est faible, mais celui des concours ne cesse d'augmenter, jusqu'à dépasser le nombre de 70 par an toutes catégories confondues. L'École ne coûte pas très cher à l'État, qui n'a qu'à payer quelques dizaines de fonctionnaires et à aménager et entretenir des locaux adaptés. Il est vrai qu'il n'y a aucune compensation à ces dépenses, l'enseignement y étant gratuit.

Les ateliers pallient sa faiblesse. Ils deviennent des «écoles particulières", comme le note en 1815 Joachim Le Breton, secrétaire perpétuel de la classe des beaux-arts de l'Institut ${ }^{3}$. L'atelier est une structure privée, extérieure à l'École, avec un fonctionnement autonome. Tout architecte peut ouvrir un atelier; il y admet les élèves qui lui conviennent. Mais son succès est aussi tributaire de ses résultats dans les concours de l'École. Chaque élève doit participer financièrement au fonctionnement de l'atelier, loyer et chauffage du local, gros matériel, ainsi qu'un émolument pour le professeur. La trésorerie de l'atelier s'appelle la masse. Mais la dépense reste faible. De plus, la réforme de l'École initiée par Eugène Viollet-le-Duc en 1863, qui met fin à son système d'autogestion et la place désormais dans les conditions normales du droit public, prévoit la création d'ateliers dits "officiels» dans l'enceinte de l'École, où les patrons sont rémunérés par l'État. Les élèves n'ont plus en charge que l'achat de leur petit matériel (tés, compas, papier, encre...). Il y a trois ateliers officiels pour l'architecture.

C'est le professeur de théorie de l'architecture qui, au-delà des cours qu'il doit dispenser, a la lourde tâche de concevoir les programmes des concours donnés mensuellement à l'École. Mais c'est dans l'atelier que l'élève apprend à dessiner et composer, la base de l'enseignement de l'architecture à l'École. La prédominance de la composition d'architecture sur les connaissances techniques s'est peu à peu imposée. C'est au

2. B. Bergdoll, Les Vaudoyer, une dynastie d'architectes, p. 19 et 26.

3. J. Lebreton, Notice des travaux de la classe des Beaux-Arts de l'Institut royal de France depuis le mois d'octobre 1814, p. 15 sq. 
point que Julien Guadet, qui, après avoir tenu un atelier à partir de 1871, devait prendre en charge en 1894 la chaire de professeur de théorie, avance en 1882 avec le plus grand sérieux qu'on peut se passer d'enseigner les matières scientifiques à l'École dans la mesure où on peut aller les acquérir ailleurs, et écrit en toute certitude:

L'atelier, qu'il soit dans l'École ou au dehors, est [...] l'instrument par excellence de l'enseignement artistique. De tout temps, la vraie école a été l'atelier ${ }^{4}$.

\section{Trois cent quarante élèves et plus}

Trois cent quarante élèves suisses ont été admis à l'École des beaux-arts entre 1809 et 1961. Ils ont été certainement beaucoup plus nombreux. On pouvait suivre les cours de l'École en auditeur libre, à condition d'en obtenir l'autorisation, et on pouvait aussi entrer dans un atelier sans fréquenter l'École.

Une lettre écrite à la demande de la légation suisse à Paris en 1922 donne une idée de la proportion entre élèves scolarisés et éléments extérieurs ${ }^{5}$. Sur un total de 63 Suisses recensés, 19 ont été reçus à l'examen d'admission, 26 inscrits dans les ateliers, 18 acceptés dans les galeries de moulages et les salles de cours, soit un rapport de un à trois entre élèves de l'«École proprement dite», comme on appelait l'établissement de la rue Bonaparte par rapport aux ateliers dits «extérieurs» ou "libres», et les simples auditeurs, dits aussi "aspirants». Et encore, il est probable que les 26 élèves décomptés dans les ateliers n'étaient que ceux des trois ateliers officiels. Même si cette enquête fait le point sur la reprise des cours après la Grande Guerre, alors que l'École des beaux-arts, décimée et privée de ses forces vives comme la France entière, ne parviendra pas à retrouver le prestige qu'elle avait encore au tournant du siècle, elle donne à penser que les Suisses ont été trois fois plus nombreux à fréquenter l'École que ceux que nous avons recensés.

Pour compléter ces chiffres, il faut aussi se faire une idée de ce que pesait l'École des beaux-arts au sein du monde du bâtiment. Nous

4. J. Guadet, L'enseignement de l'architecture.

5. Archives nationales de France (AnF), $\mathrm{AJ}^{52}$ 909: École des Beaux-Arts, statistiques, 1876-1966. 
disposons pour ce faire du comptage auquel s'est livré en 1899, à partir des annuaires professionnels, un chef d'atelier libre, Gaston Redon (1853-1921), jeune frère du peintre Odilon Redon, alors que les membres du Conseil supérieur d'enseignement de l'École révisaient les conditions d'admission ${ }^{6}$. Sur les 6672 architectes pratiquant alors en France, 1200 seulement sont passés par l'École. Alors que la profession d'architecte n'était pas protégée - l'Ordre des architectes, qui devait imposer d'être architecte diplômé par le gouvernement pour être autorisé à signer des plans, n’a été institué que le 31 décembre 1940 -, les anciens élèves de l'École des beaux-arts représentaient seulement entre un cinquième et un sixième de l'ensemble des praticiens. On peut imaginer une situation assez similaire en Suisse.

Les élèves sont majoritairement originaires de la partie francophone de la Confédération. Deux cent quarante-six viennent de la ville et du canton de Genève, du canton de Vaud et, dans une moindre mesure, de celui de Neuchâtel. Mais la Suisse germanophone n'est pas si mal représentée, avec 93 ressortissants, dont 24 venus du canton de Zurich. La seule exception notable est l'absence quasi totale de la partie italophone du pays, avec un seul élève né au Tessin, Lorenzo Lepori (1789-1853), et, beaucoup plus tard, le Lausannois Louis Brazzola (1875-1948) qui n'en est qu'originaire ${ }^{7}$.

\section{Quelques pics de fréquentation}

Le graphique de la fréquentation des élèves en architecture suisses à l'École des beaux-arts laisse entrevoir quelques pics: 1846, 1857, 1862, $1876-1884$, autour de $1900 \ldots$ Toutes ces dates ne recouvrent pas forcément des réalités économiques et politiques spécifiques, car on voit

6. Conseil supérieur d'enseignement de l'École des beaux-arts, procès-verbal de la séance du 24 avril 1899 ( $\mathrm{AnF}, \mathrm{AJ}^{52} 20$ ).

7. On y ajoutera une douzaine de natifs d'autres régions dont les noms sont d'origine italienne. Ce chapitre mériterait une étude plus approfondie. On notera seulement qu'il n'est pas toujours évident de définir l'origine exacte de chacun, d'autant que beaucoup sont issus de couples mixtes. D. Lüthi (La construction de l'architecte, p. 81 sq.) a remarqué qu'il y avait à La Chaux-de-Fonds une importante communauté de maçons et d'entrepreneurs originaires du Tessin et de l'Italie. 
régulièrement des Suisses venir en France pour quelques années ou plus, en quête d'un marché plus porteur et formateur.

Nous nous arrêterons sur l'année des premiers succès, 1846. La Suisse connaît alors une période de crise économique et politique, qui débouche sur la guerre du Sonderbund en 1847, et la constitution fédérale de 1848 qui fonde la Suisse moderne ${ }^{8}$. Quelques architectes s'expatrient alors en France, comme Jean Baptiste Bertolini (1822-1883), venu en 1846 à Paris où il travaille notamment chez Hector Lefuel et participe au chantier du Louvre avant de s'installer à Lausanne, associé à Jules Carrard (1833-1906), élève de Lebas admis à l'École en 1857, ou JulesLouis Verrey (1822-1896), élève de Labrouste non admis, qui exerce pendant une quinzaine d'années à Melun en Seine-et-Marne, avant de venir se fixer à Lausanne et d'y fonder en 1861 la plus ancienne agence d'architecture du canton de Vaud.

En 1846, cinq étrangers, dont quatre Suisses, David Braillard (18261896), Johann Carl Dähler (1823-1890), Jean Jacques Stehlin (18261894) et Jean Franel (1824-1885), sont admis à l'École. Le cinquième, Richard Morris Hunt (1827-1895), premier Américain de souche admis à l'École des beaux-arts, n'est pas loin d'être un Suisse!9 Placé par sa mère dans une pension tenue par Alphonse Briquet (1809-1880), descendant d'une famille réformée de Châlons-sur-Marne émigrée à Genève vers 1724 pour cause de religion ${ }^{10}$, Hunt entre chez l'architecte genevois Samuel Darier (1808-1884), un ancien élève de Huyot à l'École des beaux-arts qui a ouvert son cabinet en 1837 et l'oriente comme un autre

8. Je remercie Dave Lüthi pour tous les repères chronologiques et les informations qu'il m'a fournis.

9. Sur ses années de formation: P. R. Baker, Richard Morris Hunt, p. 13-44; S. Stein, The Architecture of Richard Morris Hunt, p. 13-45.

Io. La pension Briquet accueille 250 élèves, parmi lesquels de nombreux étrangers, Français, Anglais, une vingtaine d'Américains. Ernest Burnat (1833-1922) y sera pensionnaire en 1848, avant d'être lui aussi admis à l'École en 1855 , comme élève de Guénepin. Le fils de Briquet, Émile (1835-1889), sera pour sa part diplômé de l'École Centrale des arts et manufactures à Paris en 1857, avant de diriger une fabrique d'appareils de chauffage en association avec Jules Weibel (1834-1886), diplômé de Centrale en 1856, et père de l'architecte Charles Weibel (1866-1942), élève de Gerhardt admis à l'École des beaux-arts en 1887. 


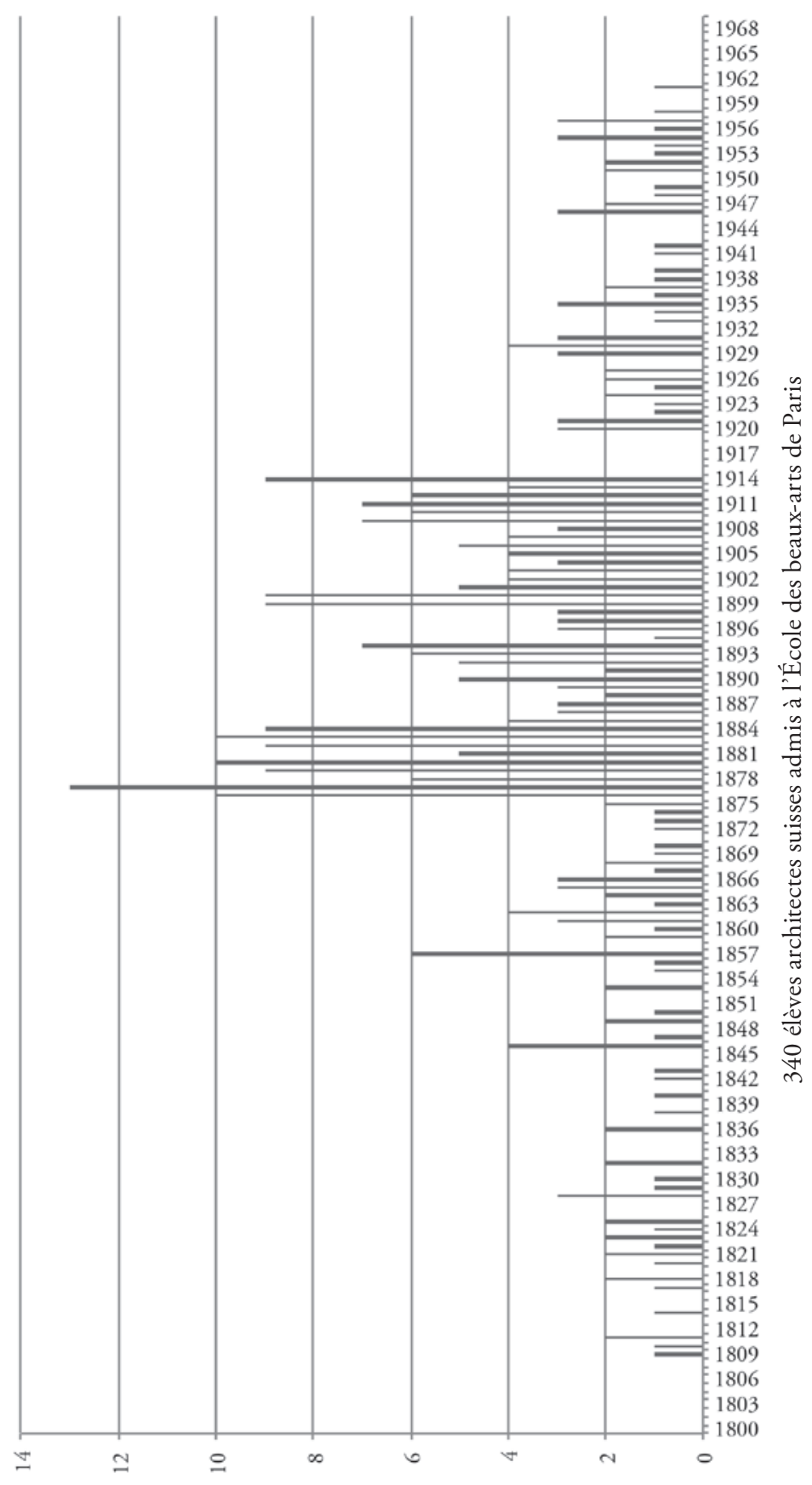


de ses jeunes collaborateurs, Jean Franel (1824-1885), vers un de ses anciens camarades à l'atelier, Hector Lefuel ${ }^{11}$.

Cette année 1846 a-t-elle constitué un phénomène isolé ou marque-telle une date pivot dans l'attractivité exercée par l'École de Paris sur les candidats architectes venus de Suisse? Et le rôle joué par cette pension genevoise et par Darier a-t-il été épisodique ou déterminant? Nous ne faisons que lancer quelques pistes de réflexion...

De l'École centrale des arts et manufactures (Paris, 1829) à l'École spéciale de Lausanne (1853)

La comparaison avec l'École Centrale des Arts et Manufactures est particulièrement instructive ${ }^{12}$.

Entre 1829, date de la fondation de l'École centrale, et 1878, 4139 élèves sont sortis diplômés, dont 891 étrangers. Parmi eux, 114 Centraliens sont nés en Suisse. Dans la mesure où un quart des élèves sort diplômé, on en conclura que l'hôtel Salé - aujourd'hui musée Picasso -, qui abritait dans le Marais l'École Centrale, a accueilli environ 400 élèves suisses. Deux font partie de la première promotion, Jean-Louis Étienne Du Pan né à Genève et Jacques Veret né à Nyon. Le maximum du contingent suisse est atteint entre 1848 et 1858 , avec entre quatre et neuf élèves diplômés par an. La création d'écoles d'ingénieurs en Suisse tarira ce flux migratoire. Les Suisses qui figurent dans l'annuaire de Centrale en 1938 ne sont plus que six.

Dans la même tranche chronologique, ce sont seulement 86 élèves nés en Suisse qui sont admis à l'École des beaux-arts. S'il n'y a aucun diplômé, c'est que le diplôme n'est organisé qu'à partir de 1869, et que le premier diplômé suisse l'est en 1881 .

II. Franel construira le Conservatoire de musique en 1856-1858 sur les plans de l'architecte français Jean Baptiste Lesueur, en collaboration avec Darier.

I2. Le cas des élèves suisses de l'École polytechnique a été étudié par Paul Bissegger («Étudiants suisses à l'École Polytechnique de Paris (1798-1850)»). Mais l'embrigadement de Suisses dans les armées napoléoniennes en fait un cas spécifique. En revanche, celui de l'École Centrale reste à étudier: il n'a fait l'objet que de trois petites pages par Jean-Louis Bordes («La Suisse et l'École Centrale»). Je n'en remercie que plus JeanFrançois Belhoste, ancien centralien et directeur d'études à l'École Pratique des Hautes Études, de m'avoir fourni les informations qui suivent. 
Précisons cependant que, contrairement à Centrale, la sélection aux beaux-arts se fait à l'entrée, et que l'examen d'admission y est très difficile, non que les épreuves soient particulièrement complexes ni le niveau des concurrents excellent, mais parce que l'offre en places d'élèves est mince au regard du nombre d'aspirants. L'écrémage ne fait que s'alourdir au fil des promotions. À la session d'avril 1878, 115 esquisses sont rendues à l'épreuve d'architecture et, à l'issue du concours, 51 concurrents sont admis. Vingt ans plus tard, à la session de printemps 1899, où un numerus clausus est fixé pour la première fois à 45 Français et 15 étrangers, 357 concurrents ont rendu une esquisse.

Le système de sélection entre les deux écoles n'explique pas un tel écart de fréquentation. Les Suisses sont plus attirés par le métier d'ingénieur que par celui d'architecte. Désenclaver un pays montagneux en traçant voies carrossables et ferrées nécessite des ingénieurs capables de construire à flanc de montagne des ouvrages d'art qui atteignent souvent une dimension héroïque. En même temps, le marché de l'immobilier reste longtemps amorphe, les architectes se disputent un marché limité à quelques rares propriétaires fortunés et doivent élaborer des stratégies pour approcher cette clientèle ${ }^{13}$.

\section{Des origines sociales sensiblement différentes}

Cette dernière considération amène à s'interroger sur l'origine sociale des Suisses venus étudier l'architecture à Paris. Ce sont généralement des jeunes gens de bonne famille, parfois aristocratique, assez aisés pour aller étudier à l'étranger. Ils font plutôt des carrières honorables, plusieurs s'investissent en politique. Les administrations distribuent rarement des bourses, et quand elles le font, c'est tardivement, à partir des années 1890 seulement.

Leurs camarades français, plus jeunes, sortent juste de l'école ou du lycée. Ils sont majoritairement d'origine modeste, fils d'artisans, ouvriers, couturières, et même serviteurs. Le père de Jean-Louis Pascal (1837-1920), un des patrons d'atelier préférés des Suisses, était cuisinier. Rares en France sont les parents de profession libérale, plus rares encore les ascendances nobiliaires. Il n'est pas de bon ton pour un aristocrate

13. D. Lüthi, La construction de l'architecte. 
de gagner sa vie en travaillant. Malgré la gratuité des études, la vie à Paris est chère pour des étudiants pauvres, mais les talents prometteurs sont subventionnés par leur ville ou leur département, et l'école de la République joue bien son rôle d'ascenseur professionnel et social.

Très vite, des familles d'architectes se forment en France sur deux, trois, quatre générations ou plus, et c'est une profession libérale respectée. Des dynasties de maîtres d'œuvre existent aussi en Suisse depuis longtemps. L'aristocratie des architectes se constitue en France avec les Prix de Rome qui ont en charge les palais de l'État et les grands bâtiments civils. Dans un contexte où l'administration est centralisée et puissante, et où le marché de l'immobilier connaît de fortes dynamiques dans le courant du XIX ${ }^{\mathrm{e}}$ siècle, faire de la politique n'apparaît pas en France comme une nécessité, comme elle l'est en Suisse pour se rapprocher des hommes de pouvoir qui sont aussi des commanditaires potentiels. La profession s'exprime en France beaucoup par la voix des sociétés d'architectes.

Les différences d'origine, de niveau social, de religion ont certainement suscité des chocs culturels entre camarades français et helvètes, mais ces derniers semblent avoir été peu diserts sur leurs expériences parisiennes, au contraire des Américains, dont les journaux et correspondances, collections et archives, nous permettent souvent d'en savoir beaucoup plus sur l'École des beaux-arts que de leurs camarades français qui la vivaient au quotidien.

\section{Double formation?}

Les Suisses commencent souvent leurs études dans des écoles et des universités allemandes ou suisses avant de partir compléter leur éducation à Paris. On trouve des cas d'élèves de Lausanne et de Zurich, diplômés dès les années 1850 , qui partent ensuite à Paris. Le processus ne se fait pratiquement jamais en sens inverse et les études antérieures à la venue à Paris sont plusieurs fois, dans la lettre produite par la légation suisse à Paris pour la demande d'inscription à l'École, qualifiées d'«études préliminaires». Les beaux-arts semblent bien être regardés en Suisse comme un établissement d'enseignement supérieur, où on vient se perfectionner par l'acquisition des règles de composition et la maîtrise du dessin, qui restent d'un faible niveau dans les écoles d'ingénieurs. 


\section{Diplôme DPLG, double diplôme?}

Les Suisses sont généralement mieux formés pour l'examen d'admission que les Français, qui sortent à peine de leurs études secondaires. S'ils ne semblent pas réussir mieux que les autres à passer l'admission du premier coup, onze d'entre eux sont classés premiers de leurs promotions.

En revanche, si on considère le déroulement du cursus scolaire, il apparaît que les Suisses poursuivent le plus souvent leurs études a minima, sans chercher à obtenir des résultats spectaculaires. La bibliothèque de l'École ne conserve que quelques dessins de concours d'élèves suisses ${ }^{14}$. Les cursus d'exception, sanctionnés par la Grande Médaille d'émulation qui récompense les meilleurs succès scolaires, ou le prix du meilleur diplôme, se réduisent à cinq cas.

Il y a une génération, entrée à l'École des beaux-arts entre 1876 et 1884, qui fait montre de solides qualités techniques, qui lui permettent de remporter dans la décennie 1880 quatre fois le prix Jaÿ pour le meilleur projet de construction, le concours considéré comme le plus difficile de l'École, et qui, obligatoire pour pouvoir monter en première classe, demande souvent un an de préparation: Eugène Schaltenbrand en 1881, Henri Mauerhofer, diplômé de Lausanne, en 1882, Augustin ReySpitzer en 1884, Maurice Vallat fils, ancien élève de l'École d'horlogerie de Neuchâtel, en 1887. Ils poursuivront leurs études jusqu'au diplôme, à l'exception de Vallat. Mais les résultats des Suisses en composition d'architecture semblent moins convaincants, indice d'une formation antérieure plus faible dans ce domaine. Au total, 139 Suisses sont allés jusqu'au diplôme. Mais ils s'y sont mis sur le tard, à partir des années 1900, quand il était devenu inimaginable de ne pas aller au bout de ses études. Et les cas de double diplôme sont rares; nous n'en avons identifié que cinq.

I4. Les élèves classés premiers étaient tenus de déposer leurs dessins à la bibliothèque de l'École. Cf. le catalogue en ligne des collections de l'École des beaux-arts: <http://www.ensba.fr/ow2/catzarts/index.xsp>. On n'y retrouve guère que les signatures d'Henri Chessex, Alfred Aubert, Eugène Schaltenbrand, Charles Schulé, Eugène Jost, Maurice Vallat et Hermann Neukomm. Les publications consacrées aux concours de l'École élargissent un peu le corpus. 


\section{Les ateliers les plus fréquentés}

Quel atelier choisir? On a beaucoup parlé de préférences particulières des Suisses pour certains ateliers, notamment celui de Pascal. Le dénombrement des élèves admis à l'École, atelier par atelier, et la comparaison, nationalité par nationalité, révèle des choix plus diversifiés. Nous ne pouvons ici qu'évoquer les ateliers les plus importants.

L'atelier Pascal a pris la suite de celui de Charles Questel, de même que Victor Laloux a été l'assistant de Louis Jules André, avant d'être choisi par une majorité de ses élèves comme son successeur. Julien Guadet dirige un atelier officiel, avant d'être nommé professeur de théorie en 1894. Latelier Henri Deglane et l'atelier collégial de Ginain, Blondel et Scellier de Gisors sont des ateliers libres.

La structure des ateliers est extrêmement variable, tant dans leur durée que dans leur importance numérique. Ils dépendent de leur attractivité auprès des élèves, mais à l'inverse, la présence des étrangers semble dépendre de l'intérêt que les professeurs leur accordent. Guadet se réjouit en 1882 d'avoir dans son atelier un étranger sur quatre ou cinq élèves, dont "des Suisses en grand nombre» ${ }^{15}$. Vingt ans plus tard, Laloux, dont l'atelier attire tout particulièrement les Américains, n'hésite pas à se servir de ses élèves les plus talentueux pour le valoriser, retenant pendant trois ans après son diplôme en 1901 le Californien Arthur J. Brown, Jr. (1874-1957), afin qu'il continue jusqu'à ses trente ans à récolter des récompenses, "négrifie» pour ses camarades candidats au Grand Prix, et même étudie en tête à tête avec lui les programmes supposés devoir être donnés au Concours de Rome ${ }^{16}$. Pascal pour sa part vitupère contre ces mêmes Américains qui viennent fréquenter en touristes son atelier pour six à huit mois ${ }^{17}$.

Il est difficile de faire une comparaison fine entre des ateliers dont les périodes d'activité se chevauchent mal sur le plan chronologique. En se limitant à la période 1870-1914, qui correspond aux années de plus forte fréquentation des Suisses, on constate que l'atelier André-Laloux comptait plus du double des élèves de Pascal, une même proportion

I5. J. Guadet, L'enseignement de l'architecture, p. 7.

I6. J. T. Tilman, Arthur Brown Jr., p. 30 sq.

17. Pascal à Paul Cret, 25 octobre 1903 (Archives of the University of Pennsylvania, Paul Philippe Cret collection, carton 8, dossier 340). 
d'Américains (et pourtant Pascal parlait anglais), et à peine moins de Suisses. La préférence quavaient les Suisses pour Pascal tenait-elle simplement au fait qu'il avait succédé à Questel, atelier où ils avaient déjà leurs habitudes? Il est certain que Questel, puis Pascal ont assez marqué les mémoires pour que les principales revues d'architecture suisses leur consacrent une notice nécrologique à leur décès ${ }^{18}$. Les qualités de grand dessinateur et pédagogue de Pascal, ainsi que sa probité et sa bonté, ont certainement eu leur part dans ces choix. Était aussi appréciée sa vaste connaissance des styles anciens, qui s'est particulièrement manifestée dans le respect qu'il a eu à la Bibliothèque nationale pour les constructions anciennes sur lesquelles il devait se greffer, contrairement à Labrouste. Mais on conviendra que ce type de qualités attire tous les élèves. On avancera que les Suisses étaient peut-être plus sensibles au contenu de l'enseignement qu'aux succès scolaires, motivation majeure des Américains pour intégrer l'atelier Laloux. Il nous manque pour affiner ces appréciations des notes plus personnelles et moins convenues que l'exercice obligé que sont les rubriques nécrologiques.

Latelier Pascal se situait au 20, rue Mazarine, derrière l'Institut de France, à proximité de la rue Bonaparte. L'esprit de corps y était extrêmement fort, en témoigne un joli petit livre offert à Pascal en 1897 à l'occasion des vingt-cinq ans de son atelier ${ }^{19}$. Ses plus talentueux aquarellistes, parmi lesquels Frédéric de Morsier (1861-1931), ont décoré chacun une page sur laquelle sont portés les noms des élèves de l'atelier. Dix ans après sa mort, le beau profil en médaille de Pascal veillait encore sur l'atelier, alors dirigé par Duquesne, Nénot et Recoura.

\section{La présence étrangère contestée}

Il faut aborder un sujet délicat, une présence étrangère jugée de plus en plus pesante. La France de Napoléon III a subi une cuisante défaite contre la Prusse en 1870. Elle ne retrouvera son honneur qu'en 1918, et la population, toute à sa rancœur, ne fait pas toujours la différence entre Allemands et Suisses alémaniques. De plus, l'armée française ayant été

18. Pour Pascal: Edgar Schlatter in Bulletin technique de la Suisse romande, 47, 26, 1921, p. 311 sq., et Schweizerische Bauzeitung, 78, 23, 3 décembre 1921, p. 280 sq.

I9. Bibliothèque nationale de France, Cabinet des estampes, BNFest Pd122 rés. 
jugée mal préparée, une loi de 1872 rend le service militaire obligatoire. Quand l'âge limite des études est de trente ans, c'est une décision qui pèse lourd: les Français ne luttent pas à armes égales avec les étrangers, qui bénéficient de l'exemption. Venus à Paris en nombre, ceux-ci participent de plus de manière notable à l'encombrement des ateliers, qui ne peuvent accueillir les élèves de première et de seconde classe qu'en alternance.

Une brochure publiée par un journaliste vers 1886 témoigne du malaise des élèves, qui pétitionnent pour "limiter le nombre des places accordées aux Anglais, aux Américains et surtout aux Suisses allemands, dont ces ateliers regorgent ${ }^{20}$. Ils voient les prix de fondations, rémunérés en argent, leur échapper au profit de concurrents plus mûrs et mieux préparés qu'eux. Le prix Jaÿ de construction est particulièrement pointé, qui "paraît tomber régulièrement aux mains de quelque étranger, de tel Suisse par exemple, élève-ingénieur de l'École de Zurich, venu à Paris pour ajouter à son diplôme celui d'architecte de l'École des beaux-arts de France. Toute la partie technique de la construction étant comprise dans ce qui constitue la science de l'ingénieur, ces Suisses, qui sont des hommes faits, ont un premier avantage sur les jeunes Français de seconde classe [...]; et cette avance, jointe à cet autre avantage de l'exemption militaire, leur procure dans les années de l'École des facilités tout exceptionnelles pour obtenir ces prix tant recherchés par les jeunes gens sans fortune " ${ }^{21}$. Voilà qui montre au moins que l'École de Zurich est, en une vingtaine d'années, arrivée à maturité et s'avère une concurrente redoutable.

Les jeunes Français savent aussi qu'ils ne peuvent pas trop compter sur l'appui des anciens, qui sont installés, ni de leurs professeurs, flattés d'attirer à eux des étrangers - on l'a vu avec Guadet -, et ils constatent que les jurys font facilement preuve de mansuétude envers eux, pensant qu'ils leur feront une bonne réputation hors des frontières. Or, la Suisse a connu dans les années 1870-1880, avec la Grande dépression, un ralentissement des chantiers. Ses concitoyens viennent aussi en France pour chercher du travail, un phénomène qui semble se reproduire au tournant du siècle. Ils se font naturaliser, mais pas trop tôt, afin d'échapper à la conscription, et peuvent ainsi s'insérer plus tôt dans le marché du travail.

20. L. Verax, De l'envahissement de l'École des beaux-arts par les étrangers, p. 6.

2I. Id., p. 7. 
Enfin, la France, fière d'attirer à elle toutes les nations du monde, est un pays ouvert, et Paris est la seule capitale européenne à avoir connu récemment un accroissement de sa population étrangère de $5 \%$.

Elle est pourtant en butte au protectionnisme ambiant: les Américains taxent à $33 \%$ les importations d'œuvres d'art, les Italiens ont privé, du fait qu'il était français, Henri-Paul Nénot de l'exécution du monument à Victor Emmanuel à Rome, dont il avait été le lauréat en 1882, les Suisses font payer aux étrangers une taxe de séjour. On peut s'interroger sur le retour d'investissement pour la France d'une politique d'accueil qui se révèle plus coûteuse que rentable.

Il semble aussi que sitôt rentrés chez eux, les Suisses se soient très vite mis aux affaires et n'aient gardé de leur passé scolaire qu'un vague esprit de corps et certaines tournures d'esprit, dont témoignent quelques notices nécrologiques. L'Association des anciens élèves de l'École des Beaux-Arts de Paris, Architectes suisses ou résidant en Suisse, ne semble pas avoir eu une durée de vie bien pérenne.

Quant aux concours publics organisés en Suisse, ils étaient le plus souvent verrouillés, réservés aux ressortissants du canton ou du district où ils étaient organisés, comme en témoignent les règlements publiés dans la revue française, Les Concours publics d'architecture, par l'architecte Laurent Farge, entre 1895 et $1914^{22}$.

Autant la France semble avoir été une terre d'accueil pour les architectes et ingénieurs suisses, autant la Suisse semble s'être cloisonnée à l'intérieur de ses frontières...

Marie-Laure Crosnier Leconte Institut national d'histoire de l'art, Paris

22. Cf. M.-L. Crosnier Leconte, «Les grands concours internationaux (1895-1914)». 


\section{BIBLIOGRAPHIE}

Baker, Paul R., Richard Morris Hunt, Cambridge, Mass., M.I.T., 1980. Bergdoll, Barry, Les Vaudoyer, une dynastie d'architectes, catalogue d'exposition, Paris, Musée d'Orsay, 1991.

Bissegger, Paul, «Étudiants suisses à l'École Polytechnique de Paris (1798-1850)», Revue suisse d'histoire, 39 (1989), p. 115-151.

Bordes, Jean-Louis, "La Suisse et l'École Centrale», Centraliens, 568 (2006), p. 56-60 (en ligne: <http://centrale-histoire.centraliens. net/pdfs/revues/rev568.pdf $>$ ).

Crosnier Leconte, Marie-Laure, "Les grands concours internationaux (1895-1914): vecteurs parallèles de diffusion de l'architecture française?", in Repenser les limites: l'architecture à travers l'espace, le temps et les disciplines, Paris, INHA, 2005, en ligne sur <http:// inha.revues.org/454>.

Guadet, Julien, L'enseignement de l'architecture. Conférence faite à la Société centrale des architectes le 24 mars 1882, Paris, Ducher et $\mathrm{C}^{\mathrm{ie}}, 1882$.

Lebreton, Joachim, Notice des travaux de la classe des Beaux-Arts de l'Institut royal de France depuis le mois d'octobre 1814, Paris, Firmin-Didot, 1815.

Lüthi, Dave, La construction de l'architecte. Histoire d'une profession en Suisse romande 1800-1940, Neuchâtel, Alphil, 2010.

Stein, Susan, The Architecture of Richard Morris Hunt, Chicago/London, The University of Chicago Press, 1986.

Tilman, Jeffrey T., Arthur Brown Jr. : Progressive Classicist, New York, W. W. Norton \& Cy, 2006.

Verax, L., De l'envahissement de l'École des beaux-arts par les étrangers, réclamations des élèves français, Paris, Librairie des Imprimeries réunies, s.d. [1886]. 